\title{
INVESTIGATING THE INFLUENCE OF THE DECOY EFFECT IN PAIRWISE COMPARISON IN TERMS OF IDEA SELECTION IN THE PRODUCT DEVELOPMENT PROCESS
}

\author{
Tanaiutchawoot, Narucha (1); Bursac, Nikola (2); Rapp, Simon (1); Albers, Albert (1) \\ 1: IPEK: Institute of Product Engineering, Karlsruhe Institute of Technology (KIT); 2: TRUMPF \\ $\mathrm{GmbH}+\mathrm{Co} . \mathrm{KG}$
}

\begin{abstract}
Many activities in the new product development requires the decision making to find the final solution from multiple alternatives and make an evaluation. Even methods to support decision maker are available, the decision can go to the wrong direction because of heuristics. "Decoy effect" is a heuristic that appears in a comparison when 2 of 3 alternatives are similar but different in quality. The alternative that is similar but better in quality is possibly selected. The paper aims to understand the decoy effect by investigating it in the pairwise comparison that is a powerful technique for comparing alternatives. In an experiment, 3 ideas for the next generation of apple peeler are compared in pairs with different sequences. An impact of the decoy alternative on the comparison between other two alternatives, is investigated. Results show low impact of the decoy effect in the pairwise comparison, but this effect induces a high chance of selecting the decoy alternative when comparing the results from this study and the previous study by proposing 3 alternatives in the same sequence. Applying pairwise comparison to avoid decoy effect is thus an idea that will be further investigated.
\end{abstract}

Keywords: Decision making, Decoy effect, Pairwise Comparison, New product development, Evaluation

Contact:

Tanaiutchawoot, Narucha

Karlsruhe Institute of Technology

IPEK: Institute for Technology

Germany

Narucha.Tanaiutchawoot@partner.kit.edu

Cite this article: Tanaiutchawoot, N., Bursac, N., Rapp, S., Albers, A. (2019) 'Investigating the Influence of the Decoy Effect in Pairwise Comparison in Terms of Idea Selection in the Product Development Process', in Proceedings of the 22nd International Conference on Engineering Design (ICED19), Delft, The Netherlands, 5-8 August 2019. DOI:10.1017/dsi.2019.125 


\section{INTRODUCTION}

"Decoy effect" or "asymmetric dominance" is the phenomenon that decision makers tend to have a specific change in preference between two alternatives when a third alternative is presented. If two alternatives are presented, people tend to select an option based on their preference. If a third alternative that is similar to one of the previous two alternatives - but of lower quality, the higher quality option becomes dominant and more appealing. This third option is called the "Decoy Alternative". This is the result of cognitive biases-error in thinking that affects decisions and judgment based on memory or how you recall an event. Influence of the decoy effect is particularly strong when presented options relate to price and benefit. Many examples about decoy effect can be seen from studies in economic behavior and marketing such as subscribing online magazine that is described in a study by Ariely (Ariely 2008).

Excessive option in product development requires making decisions. This most certainly entails the use of the decoy effect. Though there are many methods and techniques to assist decision making, errors are possible due to cognitive biases from alternative structures. A rational decision can also equate to an irrational decision. In unfamiliar environments and complex situations, decision making automatically evokes intuition. This renders the probability of cognitive biases in decision making. These biases can induce wrong decision by rejecting a good solution and select a bad one. These poor decisions result in erroneous product development, thus affecting the consumption of time and budget. However, these biases are often unexplained in a product development point of view, leading to unaware decision makers. Understanding the impact of the decoy effect on product development is imperative. A study on the decoy effect, regarding the number and sequence of options, is also being analyzed. Wisely, strategies to reduce this process should be investigated and applied in an alternative prioritization and selection. In the next section, a state of the art economic study will be presented featuring an example of the decoy effect". The same effect on the product development study will be further illustrated.

\section{LITERATURE REVIEW}

\subsection{Economic behavior}

In economic researches, cognitive biases play an important role in influencing customers to buy products. Most choices come from evaluating a balance between costs and benefits (Alian Samson 2014). In the past, the rational decision theory presented by Gray S. Becker is used to describe human behavior with stable preferences and benefit optimization (Becker 1976). This implies that human behavior is predictable and reasonable. Economic decisions, on the other hand, are not always rational when made with cognitive biases. Gains and losses proposed by Kahneman and Tversky are one of the explanations about irrational decision behavior (Kahneman and Tversky 1979). Decision makers avoid taking risks in a sure gain situation and prefer to take risks in sure loss situations. This depends on the values of gains and losses. If a value of gain is high, decision makers prefer to take a risk, but if the gain is low, taking a risk is ignored. These describe by the concave model. Losses behavior have the same pattern but in the opposite direction, which is described by the convex model.

The "Decoy Effect" is also applied in marketing, in order to persuade decision makers to select an expected alternative. In Dan Ariely's study (Ariely 2008) about an annual magazine subscription, participants in a control group are offered two alternatives: $\$ 59$ for online access, and $\$ 125$ for online access and print. A third alternative, or decoy alternative, was added at $\$ 125$ for only print edition in a study group. This options clearly are irrational when comparing cost and benefit with other 2 options. Results in the control group showed that 68 participants preferred the first alternative with $\$ 59$ for online access. The other 32 participants preferred the second alternative with $\$ 125$ for online access and print edition. Results in the study group when decoy alternative was added, showed an opposite direction of selection behavior. 16 participants preferred the first alternative, and 84 participants preferred the second alternative. No selection occurred in the third alternative. The third alternative with $\$ 125$ for only print edition encourages the second alternative with $\$ 125$ for online access and print edition to be more dominant and be focused for the decision makers by providing the same price but lower in a benefit. This implies that decision makers selected alternative by comparing information from alternatives more than considering contents in alternatives themselves, which lead an appearance of cognitive biases because of alternative structures (Dhar and Simonson 2003). 


\subsection{Decision making in product development processes}

New products are developed based on existing technical systems (Albers, Albert, Simon Rapp, Clemens Birk, and Nikola Bursac 2017) because of economic reasons and risks (Feldhusen und Grote 2013). Each step in the product development process encounters many problems and situations that required decision making and assisted methods to find solutions and continue processes (Albers et al., 2016). Decisions, especially in an early phase, are thus very important to indicate a direction of developing processes. Even systematic decisions are usually used to find solutions by analyzing information and relevant factors, decisions can also be errors because of rational thinking limitations and uncertainty situations (Lipshitz und Strauss 1997). Intuitive decisions, fast and simple processes, are substantially used based on heuristic techniques. Albar illustrated the advantages of the heuristic decision in the early phase of product development using Fast \& Frugal heuristic (Albar, F. M., \& Jetter, A. J. 2011). Moreover, the heuristic decision was applied to identify product crisis (Muenzberg, C., Stingl, V., Geraldi, J., \& Oehmen, J 2017).

Other research groups have an opposite perspective on heuristic decisions. They claimed that heuristics are the main cause that originated from cognitive biases and errors in the decision. Creativity and innovation in the new product design and development are decreasing or changing directions because of the cognitive biases in a heuristic decision (Mike Pinder 2017, Lockton 2012). Even in a method such as a scenario method (Erdmann et al., 2015) or the Multicriteria decision analysis (Montibeller, G., \& von Winterfeldt, D. 2015) that supports decision making, the heuristic decision can also influence decisions to be bias because of anchoring effects, overestimating and framing effects. These heuristics usually occur in alternative selection and evaluation because of the choice structure. Our pre-studies (Bursac et al., 2018) also showed decision biases in selecting the concept idea for the next generation product based on the decoy effect and the representativeness during an idea selection phase in product development processes. The first study aims to investigate the decoy effect in the idea selection activity by imitating an idea from the study of the annual magazine subscription in economic study. The participants in this study have to select the idea to further develop for the next generation of apple peeler. Two reasonable ideas, that are an Electrical Drive and a Drip Tray, were proposed in a control group. A Gasoline Engine, that was similar but of lower quality than the electrical drive, was added to be a third alternative in a study group. Complete results from 69 participants showed that 36\% selected the Electrical Drive and 64\% selected the Drip Tray in the control group. 65 participants in the study group had a different selection behavior. Percentages of selections were increasing to $46 \%$ for the Electrical Drive and decreasing to $49 \%$ for the Drip Tray. The other 5\% of participants selected the Gasoline Engine. This behavior occurred from the decoy effect (Gasoline Engine) that encouraged a similar but better alternative (Electrical Driver) to be a dominant and higher chance of selection than the absolute different alternative (Drip Tray). In the second study, the decision behavior in the idea selection activity is investigated when the persona that represents a group of customers is added instead of the decoy alternative (Gasoline Engine). The same behavior also occurred when adding a description of one persona called "Hans Hitech" in another group of participants (70 students). $56 \%$ of the participants selected an Electrical Drive and another $44 \%$ of the participants selected a Drip Tray. The participants concentrated only on information representative without considering a sample size of customers and other information, which is called representativeness heuristic (Tversky and Kahneman 1974). These biases within methods are big problems in the product development process especially when the result is selected from multiple alternatives and will be applied in the next step.

\section{PROBLEM STATEMENT AND OBJECTIVE}

Most researches focus on the influence of a decoy effect when a third alternative is presented at the same sequence including our previous study that is explained in Section 2.2. This research, however, aims to investigate the impact of a decoy effect in a pairwise-comparison technique, which is general and well-known by a systematic technique to compare alternatives in pairs to judge which alternative is preferred. The experiment is performed based on an idea that a decoy alternative encourages a similar but better alternative to be highly selected. Even this behavior is dominant when 3 alternatives are presented at the same sequence, we would like to see a possibility of an influence affected by a decoy effect when 3 alternatives are compared in pairs with different sequences. Influence of the decoy effect on the decision behavior will be investigated by presenting the decoy alternative in 
different sequences and components. Moreover, decision behavior in an alternative selection based on different structures of alternative such as a number and sequences of alternatives are compared. The analysis is included comparing results between the previous study in Section 2.2 and this study. The main research question is the following: To which extent can a sequence of the pair of alternatives between the decoy alternative and the similar and better alternative influence a decision in the pair of alternatives between two rational alternatives but different concept ideas? Then how can this situation occur?

\section{METHODOLOGY}

Participants are required to select a rough idea design for the next generation of apple peelers with respect to a reference product, which represents the early stage of idea detection in the product development process. At the beginning of this experiment, the participants watched the video that described functions of the reference product and procedures to create and classify ideas for the next generation of apple peeler. This video aims at forming the basic knowledge for product development. At the end of this video, there are 3 idea designs left, which are the Electrical Driver, the Drip Tray, and the Gasoline Engine. The participants have never seen these designs until the idea selection process starts. In this process, 3 idea designs are paired and presented in 3 sequences to the participants using the pairwisecomparison technique. Each participant selected the favorite design in each time. The design that has been selected 2 times represented the final selection. Pairs and sequences to propose alternative design are different between a control group and a study group as shown in Table 1.

Table 1. Pairs of alternatives and sequences in pairwise comparison in the control group and the study group

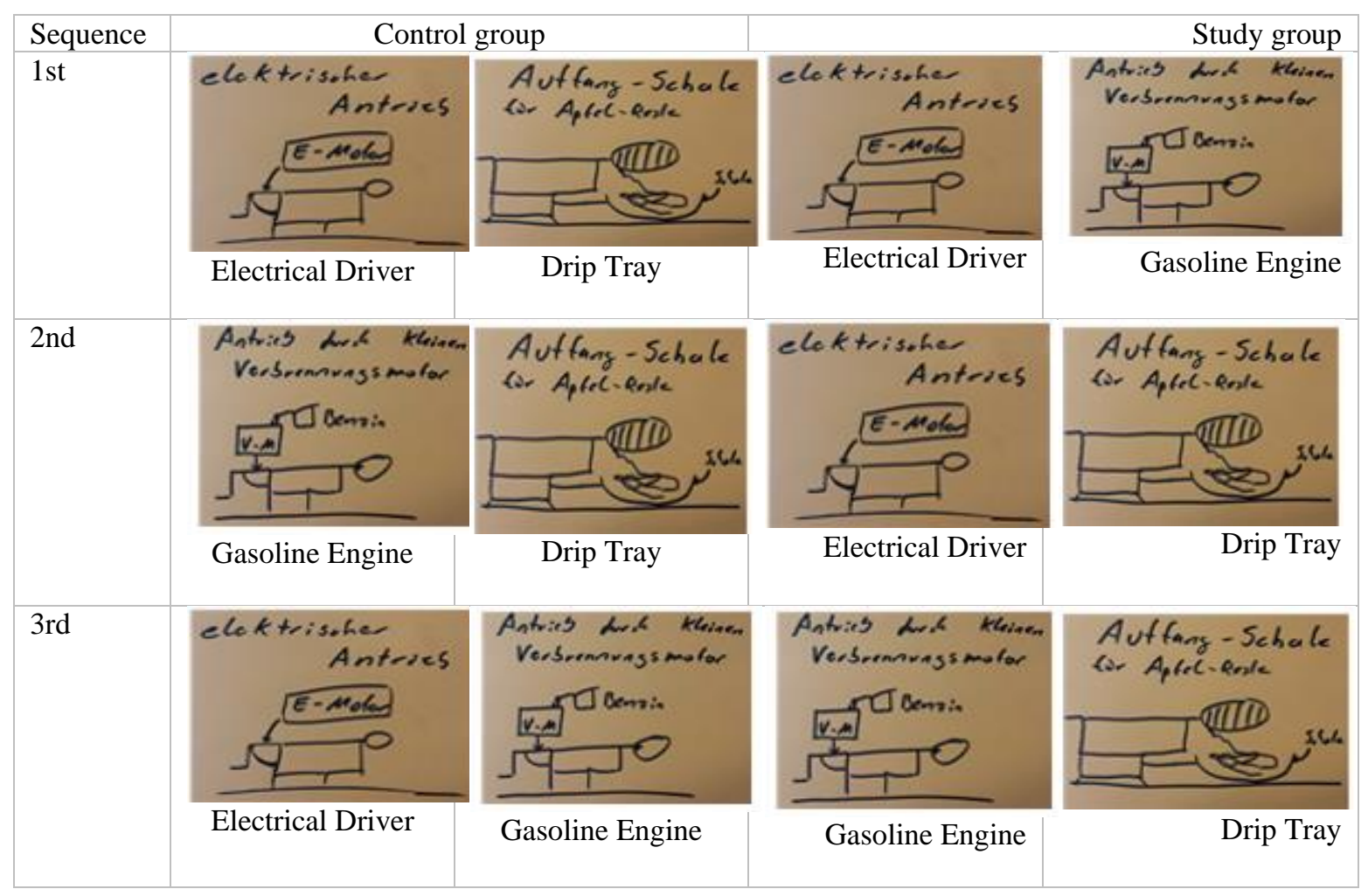

Electrical Driver and Drip Tray are assumed to have the same level of rational designs because both of them focus on solving different problems in the reference product that can be seen in the video. Gasoline Engine, on the other hand, focuses on a similar problem as the Electrical Driver, but the solution quality is lower. This design can make contamination in the apples, which shows an irrational idea design. Therefore, Gasoline Engine is assumed to be a decoy alternative to encourage the participants to focus on Electrical Driver design. In the control group, the Electrical Driver and the Drip Tray are presented for the first time in the first sequence to avoid an influence from the Gasoline Engine design or the decoy alternative. Participants have never seen the Gasoline Engine design 
before, so they will focus on selecting an idea from only the Electrical Driver and the Drip Tray. Sequences in the 2nd and 3rd comparison in this group have no meaning to investigate the decoy effect. In the study group the Electrical Driver and the Gasoline Engine alternatives are presented at the first time in the first sequence; then, the Electrical Driver (presented the 2nd time) and the Drip Tray (presented the 1st time) are proposed in the second sequence. These sequences are presented based on an idea that presenting the decoy alternative in the first sequence can affect a selection behavior in the second sequence to select the alternative that is similar but better than the decoy alternative. Even 2 alternatives are proposed to be selected in the 2 nd sequence, there are 3 alternatives in the participants' memory. Selecting idea designs in the 1st sequence in the control group and the 2 nd sequence in the study group seems to be similar to the selecting idea designs in the previous study in Section 2.2.

This study has tested the understanding of procedures and contents in a preliminary group of participants who are students of the third semester of mechanical engineering. Twenty students are separated into the half, which is ten students in the control group and another ten students in the study group. The video is presented in a lecture; questionnaires and feedbacks are answered by an online survey. After that, this study is applied in the main group of participants who are students in the 4th semester of mechanical engineering $(n=494)$. The students are divided randomly into the control group $(n=248)$ and the study group ( $\mathrm{n}=249)$. The experiment in the main study is performed via email with separating the link of the questionnaire in the control group and the study group. The survey is expired after 1 week; then the completed responding from the control group $(n=61)$ and the study group $(n=62)$ are analyzed.

\section{RESULT AND ANALYSIS}

Results from this study are analyzed in 3 steps, which have different aims and levels of investigation and analysis. These steps are described as the following:

Step 1: Results of the idea selection in the first sequence from the control group and the second sequence from the study group are compared and analyzed. The aim of the analysis step is to investigate the influence of the decoy alternative that is presented in the first sequence in the study group.

Step 2: The final results from the absolute selections of the 3 alternatives in both groups are differentiated and examined. This comparison is proposed to investigate the consistency of decision making and behavior of decision making in a pairwise-comparison technique when alternatives are presented in a different subsequence.

Step 3: The final results between the previous study in the literature review and this study are contrasted and inspected. This comparison is investigated with regard to the influence of heuristic decision based on a decoy effect in different constructions of alternatives.

All results in each study are compared and investigated with regard to a significant difference between the control group and the study group using Fisher's exact algorithm. The significant difference is selected from a one-tailed analysis to show acceptable results in comparisons.

\subsection{Results of the idea selection in the first sequence from the control group and the second sequence from the study}

The results from the participants in both groups are investigated and summarized in percentage within rounds and groups as showed in Table 2. Results of the selection between the Electrical Driver and the Drip Tray in the control group from the 1st sequence and the study group from the 2 nd sequence are then focused and compared as showed in Figure 1.

Table 2: Results of alternative selection in each sequence from the control group and the study group

\begin{tabular}{|l|l|c|c|r|r|}
\hline \multicolumn{2}{|l|}{ Order of sequences } & \multicolumn{4}{c|}{ Selection(\%) } \\
\cline { 3 - 6 } \multicolumn{2}{|l|}{ Control group (N=61) } & Study group (N=62) \\
\hline 1st & alternative & Electrical Driver & Drip Tray & Electrical Driver & Gasoline Engine \\
\hline \multirow{2}{*}{ 2nd } & $\%$ & 60.66 & 39,34 & 87.10 & 12.90 \\
\hline & alternative & Gasoline Engine & Drip Tray & Electrical Driver & Drip Tray \\
\hline 3rd & $\%$ & 27.87 & 72.13 & 43.55 & 56.45 \\
\hline & alternative & Electrical Driver & Gasoline Engine & Gasoline Engine & Drip Tray \\
\hline & $\%$ & 81.97 & 18.03 & 12.90 & 87.10 \\
\hline
\end{tabular}




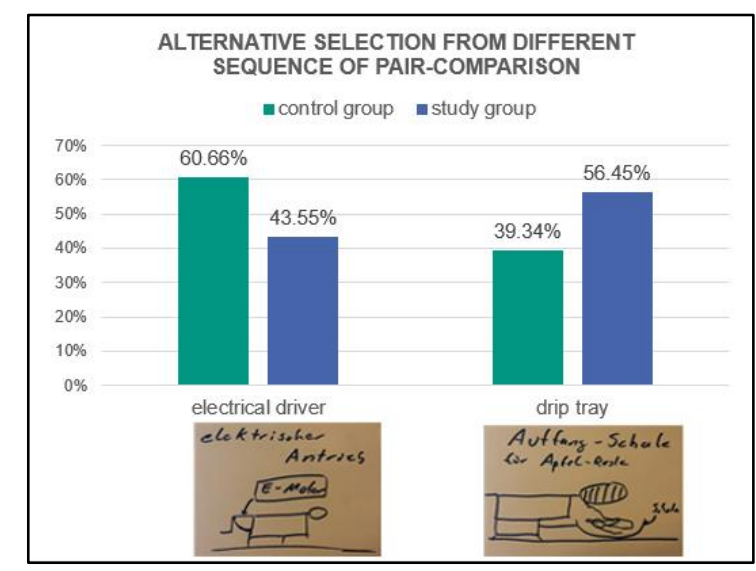

Figure 1: A comparison of alternative selections between the Electrical Driver and the Drip Tray in the control group and the study group

The results from the control group showed that $60.66 \%$ of the participants chose the Electrical Driver alternative and $39.34 \%$ of the participants chose the Drip Tray alternative. In the study group, $43.50 \%$ and 56.45\% of the participants choose the Electrical Driver and the Drip Tray alternatives respectively. The significant level of alternative selection between the control group and the study group is 0.04 . This means that there is a significant difference of $99.6 \%$ between the groups. This statistical number, however, does not show an influence of a decoy effect or an irrational idea in the alternative selection. This result is opposite to the result in the previous study from the literature review. It implies that selecting alternative using pairwise comparison can be influenced by other factors that need to be considered. However, this result illustrates our main investigation that proposing the decoy alternative with the similar but better design alternative (electrical driver and gasoline engine) before proposing the absolute different ideas (electrical driver and drip tray) does not influence decision making to select the similar but better idea. A difference in the selection behavior between both groups is not only influenced by the decoy effect but possibly results from the individual preferences of participants in each group. Other types of heuristics can also affect decision making. This is one perspective that should be further investigated.

\subsection{Final results from the absolute selections of $\mathbf{3}$ alternatives in the control group and the study group}

An individual absolute selection is calculated from finding the highest number of selection from 3 sequences. The alternative that is selected 2 times will be an absolute selection or the most preferred alternative. In the case that all alternatives are selected in different sequences, the result of that selection will be discarded because a decision in the alternative selection seems to be inconsistent. If the first alternative is selected when the first and the second alternatives are compared: This means that the first alternative is better than the second alternative. If the third alternative is selected when the first and the third alternatives are compared, the third alternative should be selected in the last comparison between the second and the third alternatives. If the second alternative is selected in the last comparison, this shows inconsistency and irrationality in decision making. One result in the study group and 2 results from the control group are discarded because of inconsistency in the alternative selection. Therefore, the absolute selections are calculated and compared from 60 participants in the control group and the other 60 participants in the study group. The absolute selections from the study group and the control group are calculated in percentage and showed in Figure 2. 


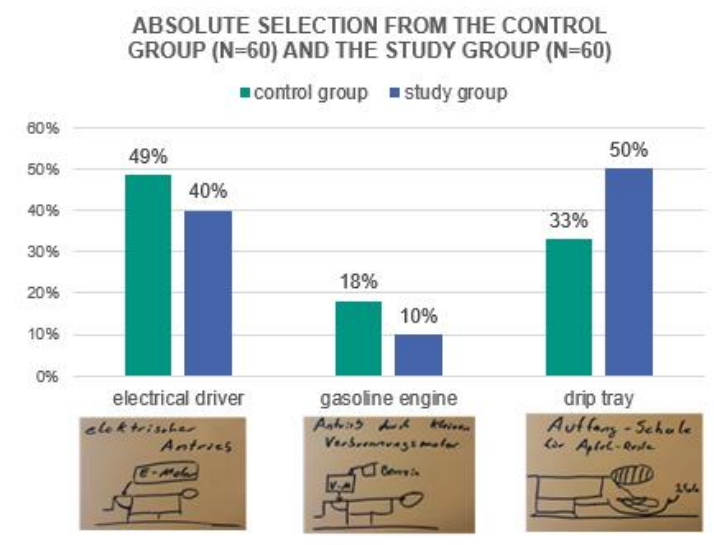

Figure 2: Final results of absolute selections from the control group and the study group

In the control group, the Electrical Driver, the Gasoline Engine and the Drip Tray are sequentially selected by $49 \%, 18 \%$ and $33 \%$ of the participants. In the study group, the Drip Tray has the highest percentage of selection with 50\%. The Electrical Driver and the Gasoline Engine were selected by $40 \%$ and $10 \%$ of the participants, respectively. The significant level of the absolute selection between both groups is 0.231 . In other words, there is a difference of $76.9 \%$ between both groups, which is lower than the previous study in the literature review $(89.1 \%)$. This result implies that proposing a decoy alternative does not influence decision behavior in the next comparison even one alternative in the next comparison is similar but better in quality while another alternative is completely different. It can be claimed that comparing alternatives that have the decoy alternative to be one of the alternatives in the sequences do not influence decision behavior. In other words, decision-making by comparing 3 alternatives using the pairwise comparison in sequences was not influenced by the decoy effect.

\subsection{Comparing the final results of the previous study in section 2.2 and this study}

The results from the previous study, that proposing 2 rational alternatives with the Electrical Driver and the Drip Tray in the control group and adding a third alternative with the Gasoline Engine in the study group, are compared and analyzed with the results from both groups in this study. Figure 3 shows the final results of both studies.

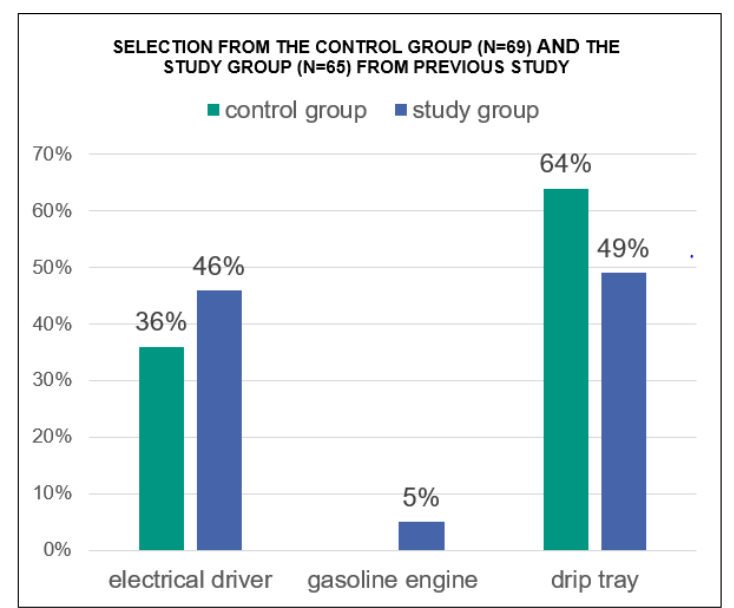

Previous study

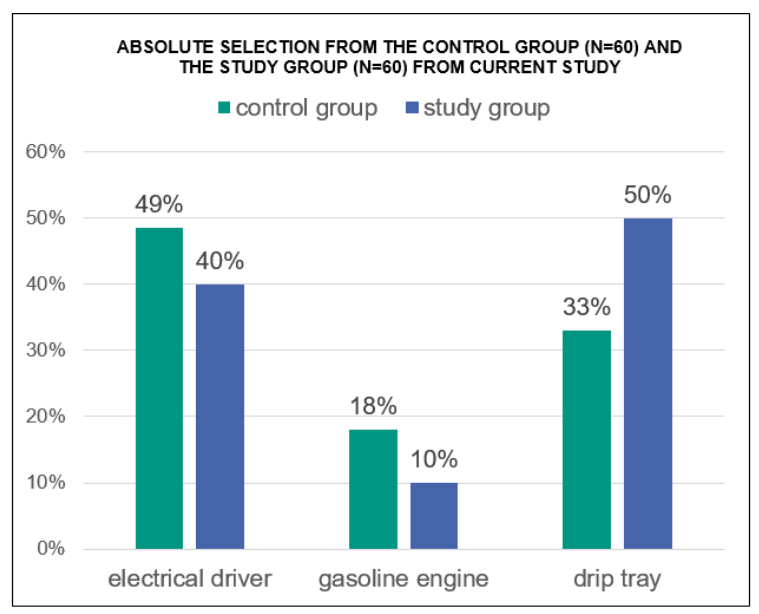

Current study

Figure 3: Results of the idea selection by the control group and the study group in the previous study in the literature review and in the current study

The behavior of idea selection in the previous study and the current study are different in the control group and the study group. In the previous study, the percentage of the participants in the control group who selected the electrical driver for further development is lower than the percentage of the participants in the study group who selected the same alternative. In the current study; on the other hand, the percentage of the participants in the control group who selected the electrical driver for further development is higher than the percentage of the participants in the study group who selected 
the same alternative. When comparing the results of the selection of the Electrical Driver between the control group and the study group in the previous study, the result shows a significant difference of 0.109 or $89.1 \%$ between both groups. When comparing the selection behavior of the study group in the current study with the control group in the previous study, the difference between both groups is $60.2 \%$ (significant difference $=0.398$ ). The percentage of differences in alternative selections between both groups in the previous study is higher than the percentage of differences in alternative selection between the study group in the current study and the control group in the previous study. The result indicates that heuristics and biases based on the decoy effect or the irrational alternative can be reduced by the pairwise comparison in sequences, which supports the conclusion in the previous comparison between the control group and the study group within the current study.

However, the percentages of the Gasoline Engine selection from the control group and the study group in the current study are higher than that of the study group in the previous study. This result shows a chance that the decoy alternative selection is higher when using the pairwise-comparison technique. Therefore, there are 2 points that should be considered from these results and analyses. Firstly, by analyzing the results from both groups and both studies in terms of electrical driver selection and gasoline engine selection, the pairwise comparison can reduce a chance of heuristic behavior based on the decoy effect to select a similar alternative that is better in quality. Secondly, the pairwise comparison possibly encourages a chance of decision making to select the decoy alternative or the irrational alternative more than proposing all alternatives in the same sequence.

\section{DISCUSSION AND CONCLUSION}

This paper aims at investigating the possibility of a decision error based on a decoy effect in the pairwise-comparison technique when the decoy alternative is paired and presented in different sequences. Even the decoy alternative can encourage a similar but better alternative to be highly selected, this effect does not occur in a pairwise comparison. This means that the decoy effect has a high impact on decision behavior by encouraging to select a similar but better alternative when all alternatives are presented in the same sequence. The decoy effect, on the other hand, has a low impact on decision making when the decoy alternative appears in a different sequence with other alternatives. This investigation provides an answer to the first research question: A sequence of the pair of alternatives between the decoy alternative and the similar and better alternative rarely influence a decision in the pair of alternatives between two rational alternatives with different concept ideas. This result can be seen as a technique to avoid and debias a decoy effect when different levels and qualities of design alternatives are compared. Instead of comparing all design alternatives in the same sequence, the design alternatives should be considered in a pair. This technique helps the decision maker to concentrate on contents of alternatives in themselves instead of comparing them to a third alternative. This synchronizes to a theory about a limitation of reasonable in decision making, which claims that people are a reason in a specific time and environment. When there is a lot of information that needs consideration, an ability to analyze information will be limited (Weick 1995). The analysis of the results of the experiment and the fundamental knowledge leads to an answer for the second research question: The pairwise-comparison technique can reduce an influence of the decoy effect by encouraging the decision maker to concentrate on the context in each alternative. The comparison between two alternatives based on the third alternative is discarded. From this advantage, the pairwisecomparison technique is a fundamental technique that is purposed to implement in a decision tool to assist the decision maker and avoid or reduce cognitive biases in the heuristic decision when the intuitive decision is applied to find a solution.

Even the pairwise-comparison technique can avoid the influence of the decoy effect when 3 alternatives are proposed, the result shows that a chance of selecting irrational alternatives is increasing. This effect has to be considered and further investigated. Moreover, results from both studies provide some hints about decision behavior related to different environments and factors of alternative structures and presentations that lead to an occurrence of other heuristics such as anchoring heuristic and the status-quo bias. This implies that the method that has the purpose of assisting and supporting the decision maker in making a decision can be influenced by cognitive bias and provide a decision error. Results in this study, therefore, originate a new idea of the study related to cognitive biases in heuristic decisions that appeared in decision making based on the pairwise-comparison technique. One question comes up with whether sequences of the alternatives influence decision 
behavior when alternatives have the same quality level? Investigating the cognitive biases in heuristic decisions based on the pairwise comparison in more detail before applying it in the decision tool is thus required.

Experiments in this paper and the previous study simulated a small model of a decision situation, which aims to describe an influence of heuristic on the decision behavior when 2 and 3 alternatives are presented and selected using different strategies. This situation can occur in product development and engineering design when alternatives or designs have a different level of quality and similarity. This behavior can also affect the decision selection when multi-alternatives that have a similarity in concept idea but different levels of quality are proposed. Decision makers cannot focus on all alternatives that are presented, brain systems will automatically manage these alternatives and reduce information by classifying, sorting and grouping alternatives to support efficiency in decision making. Moreover, people usually prefer to select alternative by comparing to a reference alternative (Kahneman 2011), which leads the result goes in a different direction based on the reference alternative. Selecting information to generate ideas and designs can be also influenced by methods that information is presented such as in sequences or coincident. The high quality of the final solution does not only rely on the number of alternatives or ideas but also relates to the process to evaluate and select alternatives. An influence of heuristic decision in general methods and situations is, therefore, important to be aware and understand to avoid and reduce decision errors and decision biases.

\section{FUTURE WORK}

Pairwise comparison is a fundamental method for Multiple Criteria Decision Making (MCDM) such as decision matrix, TOPSIS, and ANP. Therefore, understanding the effects of pairwise-comparison in decision making is important. Next step, we would like to investigate and analyze more influence factors and heuristics that possibly occur in the pairwise-comparison technique. A technique to avoid selecting irrational alternative has to be examined and then implemented. Advantages and disadvantages of this technique have to be specified. Moreover, evaluating the efficiency of this technique will be done in the real situation of product development such as idea selection and technical solution selection by comparing the results between with and without this technique. At the end of this direction, we hope a modified version of pairwise-comparison technique can be used to support decision making in the solution selection and avoid a decision bias caused by heuristics.

\section{REFERENCES}

Albar, F. M., and Jetter, A. J. (Hg.) (2011), “An investigation of fast and frugal heuristics for new project screening", Portland International Conference on Management of Engineering \& Technology (PICMET).

Albers, A., Reiß, N., Bursac, N., Breitschuh, J. (2016), "15 Years of SPALTEN Problem Solving Methodology in Product Development”. In: DS 85-1: Proceedings of NordDesign 2016, Vol. 1, Trondheim, Norway, 10th-12th August 2016.

Albers, A., Simon R., Clemens B., and Nikola, B. (Hg.) (2017), “Die Frühe Phase der PGEProduktgenerationsentwicklung”, Stuttgarter Symposium für Produktentwicklung.

Alian S. (Hg.) (2014), “The behavioral economics guide 2014”. Vol. 1. Aufl. Online verfügbar unter www.behavioraleconomics.com, zuletzt geprüft am 25,04,2017.

Ariely, D. (2008), "Predictably Irrational", The hidden forces that shape our decisions/Dan Ariely. HarperCollins, New York.

Becker, G.S. (1976), "The economic approach to human behavior”. University of Chicago Press, Chicago.

Bursac, N., Tanaiutchawoot, N., Rapp, S., Albers, A., Breitschuh, J., Eckert, C. (Hg.) (2018), "Decision heuristic in pge-product generation engineering”. TMCE 2018. Las Palmas de Gran Canaria, Spain, 7-11 May.

Dhar, R., Simonson, I. (2003), "The effect of forced choice on choice”, In: Journal of marketing research, Vol. 40 No. 2, S. 146-160.

Erdmann, D., Sichel, B., Yeung, L. (2015), “Overcoming obstacles to effective scenario planning”, In: McKinsey Quarterly, Vol. 55.

Feldhusen, J., Grote, K.-H. (2013), "Pahl/Beitz Konstruktionslehre”, Methoden und Anwendung erfolgreicher Produktentwicklung: Springer-Verlag.

Kahneman, D., Tversky, A. (1979), "Prospect Theory", An Analysis of Decision under Risk. In: Econometrica Vol. 47 No. 2, S. 263. http://doi.org/10.2307/1914185.

Kahneman, D. (2011), "Thinking, fast and slow", 1st ed. Farrar Straus and Giroux, New York.

Lipshitz, R., Strauss, O. (1997), "Coping with uncertainty", A naturalistic decision-making analysis. In: Organizational Behavior and Human Decision Processes, Vol. 69 No. 2, S. 149-163. 
Lockton, D. (2012), "Cognitive biases, heuristics and decision-making in design for behaviour change”.

Mike P. (2017), "16 cognitive biases that can kill your decision making”. Online access under https://www.boardofinnovation.com/blog/2017/08/02/16-cognitive-biases-that-kill-innovative-thinking/.

Montibeller, G., and von Winterfeldt, D. (Hg.) (2015), "Biases and debiasing in multi-criteria decision analysis", System Sciences (HICSS), 2015 48th Hawaii International Conference on: IEEE.

Muenzberg, C., Stingl, V., Geraldi, J., and Oehmen, J. (Hg.) (2017), "Identifying product development crises", The potential of adaptive heuristics. DS 87-2 Proceedings of the 21st International Conference on Engineering Design (ICED 17) Vol 2: Design Processes, Design Organisation and Management, Vancouver, Canada, 21-25.08. 2017.

Tversky, A., Kahneman, D. (1974), “Judgment under uncertainty", Heuristics and biases. In: science Vol. 185 No. 4157, S. 1124-1131.

Weick, K.E. (1995), "Sensemaking in organisation: Sage”. 